\title{
Retraction
}

\section{Retracted: Radiation Hardness of Flash Memory Fabricated in Deep-Submicron Technology}

\author{
International Journal of Photoenergy \\ Received 27 February 2018; Accepted 27 February 2018; Published 23 May 2018 \\ Copyright (c) 2018 International Journal of Photoenergy. This is an open access article distributed under the Creative Commons \\ Attribution License, which permits unrestricted use, distribution, and reproduction in any medium, provided the original work \\ is properly cited.
}

International Journal of Photoenergy has retracted the article titled "Radiation Hardness of Flash Memory Fabricated in Deep-Submicron Technology" [1]. There was a conflict of interest between the authors and its handling Editor since they share the same affiliation and have common publications. The article was reassessed by the Editorial Board, who found that the results cannot support the conclusions. The authors state "The aim of the paper is to investigate, theoretically, experimentally, and numerically, the radiation hardness of flash memory", but this was not achieved. The theoretical analysis appears to be partial, promoting a particular technological solution. Numerical and experimental investigations intended to validate the theoretical analysis are verified on few elements. Further, the effects of ionizing radiation on stored data are well known.

\section{References}

[1] B. Cavrić, E. Dolićanin, P. Petronijević, M. Pejović, and K. Stanković, "Radiation hardness of flash memory fabricated in deep-submicron technology," International Journal of Photoenergy, vol. 2013, Article ID 158792, 7 pages, 2013. 


\title{
Radiation Hardness of Flash Memory Fabricated in Deep-Submicron Technology
}

\author{
Bojan Cavrić, ${ }^{1}$ Edin Dolićanin,, ${ }^{2}$ Predrag Petronijević, ${ }^{2}$ \\ Milić Pejović, ${ }^{3}$ and Koviljka Stanković ${ }^{2}$ \\ ${ }^{1}$ Life Robinson Private Hospital, Hospital Road, P.O. Box 37, Randfontein 1760, South Africa \\ ${ }^{2}$ Faculty of Electrical Engineering, University of Belgrade, Bulevar Kralja Aleksandra 73, 11000 Belgrade, Serbia \\ ${ }^{3}$ Faculty of Electronic Engineering, University of Niš, Aleksandra Medvedeva 14, 18000 Niš, Serbia
}

Correspondence should be addressed to Koviljka Stanković; kstankovic@etf.rs

Received 5 April 2013; Accepted 25 May 2013

Academic Editor: Momčilo Pejović

Copyright @ 2013 Bojan Cavrić et al. This is an open access article distributed under the Creative Commons Attribution License, which permits unrestricted use, distribution, and reproduction in any medium, provided the original work is properly cited.

\begin{abstract}
This paper discusses the current problem of the electronic memory reliability in terms of the ionizing radiation effects. The topic is actual since the high degree of components' miniaturization integrated into the flash memory causes the extreme sensitivity of this memory type to the ionizing radiation effects. The effects of ionizing radiation may cause changes in stored data, or even the physical destruction of the components. At the end, the experimentally and numerically obtained effects of radiation on specific flash memories are shown and discussed. The results obtained by laboratory and numerical experiments showed good agreement with each other and with the theoretically expected results.
\end{abstract}

\section{Introduction}

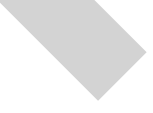

The miniaturization of electronic components includes their production by using extremely thin layers that build a composite-packaged structure capable of performing complex functions. This way of creating electronic devices makes them very irresistible to ionizing radiation in the conditions under which they operate, and in this respect, the radiation hardness of memory components is especially important [1].

The aim of the paper is to investigate, theoretically, experimentally, and numerically, the radiation hardness of flash memory. Flash memory plays an important role in the control of solar cells in cosmic application, since they are main components of devices which control all operations of solar cells in the space. In this way, the flash memory devices are exposed to intense primary cosmic radiation, so the prediction of their applicability limits is the main task of the research.

Flash memory was developed by the Japanese researcher Fujio Masuoka (1984) in the laboratories of the company Toshiba. Flash memory has an easy reprogramming ability obtained by improving EEPROM memory architecture. Flash memory bases its work on a modified MOSFET transistor with induced channel which has an insulated gate between the common gate and substrate [2,3] (Figure 1).

The operating mode of the transistor with an interrupted gate contact is determined by the preceding amount of charge that existed on the gate. This analysis is the result of the idea of the construction of a new type of the memory cell in the form of a transistor with two gates, which, besides the normal gate with a galvanic contact, also has an isolated inner gate. Based on the n-MOSFET with an isolated gate, the EPROM memory is built, after which the EEPROM memory follows, and at the end, there is flash memory.

\section{Numerical Simulation of the Radiation Interaction with Materials by Using Monte-Carlo Method}

The Monte-Carlo method is a numerical method for solving complex, mathematical, statistical, and physical problems using random selection of samples. The main goal of this method is the formation of a stochastic model which should 


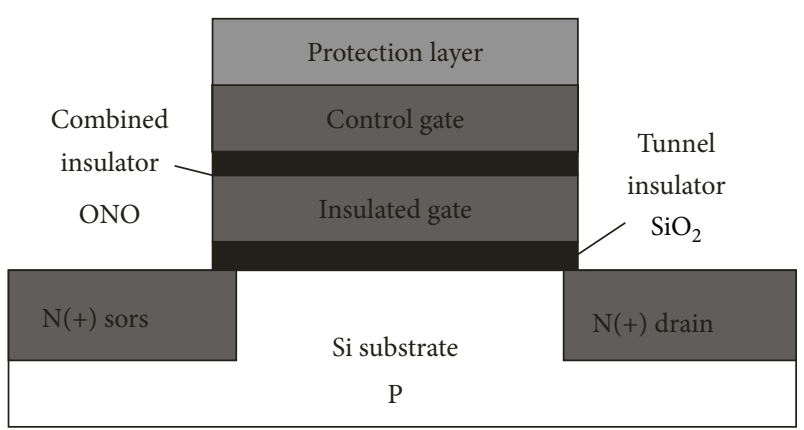

FIGURE 1: The structure of the memory cells with control (external) and insulated gate.

be consistent with the real problem or represents its direct simulation.

The basic assumption for the application of the MonteCarlo method is the possibility of generating a sequence of random numbers, that is, a sequence of independent random variables on the interval $(0,1)$. Starting from the sequence of random numbers, it is possible to generate a sequence of random distribution [4].

The simulation of passing the particles, electrons, positrons, and photons, is based on the stochastic nature of the collision processes. Between two successive collisions, a particle exceeds a random path determined by the total macroscopic cross-section and distribution density. At each collision, the particle loses energy and the energy loss is determined from the corresponding distribution density. After the collision, the particle changes its direction compared to the direction before the collision, and the angle of deviation is a random value which is selected by the angular distribution [5, 6]. On its path, the particle loses energy at the value in which considered the particle is absorbed, or with some energy leaves the medium. All collisions on the path of the particle belong to its history. By repeating the history for many times, the spatial and energy distribution of particles in the medium is obtained. The absorbed energy in the medium changes the properties of the substance and the resulting changes affect the particle passage of the "following" history because the temperature and density of the substance are changing, and in the nuclear reactions, even the isotopic composition of the substance is changed. A simple Monte-Carlo algorithm assumes that all processes are described mathematically, the transition probabilities of the particles are prepared from the previous to the next state of the phase space, an efficient algorithm of random selection of probabilities is prepared, that is an available quick and reliable model which describes the complex geometry space, and, finally, the used computer must have a reliable generator of pseudorandom numbers. When all these conditions are fulfilled, the algorithm can be translated into a computer program and simulated particles passage.

The input information required for the Monte-Carlo simulation includes

(1) dimensions of the source and the distance from the detector;
(2) dimensions of the detector housing and the sensitive and nonsensitive zones of the detector;

(3) elemental composition and density of materials through which photons and particles pass;

(4) attenuation coefficient of photons for all materials;

(5) sections for different photon interactions in the detector material as a function of energy and dispersive angle;

(6) information of the transport of electrons and positrons through the detector material.

The results of Monte-Carlo calculations agree with the measured values for the corresponding configuration, in the best case about $\pm 5 \%$. The uncertainty of the experimental value is typically less than $\pm 5 \%$, so these discrepancies must be attributed to the systematic errors or uncertainty of calculations [7].

\section{Experiment}

Experimental setting is applied to the determination of the gamma rays effect on the flash memories, which are the dominant types of long-term memory, and these memories are used in many applications that require electronic parts that work in terms of radiation.

The interaction of radiation with insulators that surround the floating gate (FG) damages the properties of memory cells, increases the possibility of losing the stored content, and damages the performance or lacks performance functionality $[8,9]$. The scope of the gamma radiation effects on the sequence of flash memory cells depends primarily on the absorbed dose. Therefore, these effects are called effects of total ionized dose (TID). The functionality of flash memories which are exposed to radiation decreases with the accumulation of TID [10].

The experiment explores the effects of gamma radiation on the cell sequence of four different flash memories. The memories are produced by different manufacturers, and all of them are currently available on market. The obtained results are interpreted in terms of the interaction of gamma rays with the internal structure of memory cells. The tested samples of memory are also compared with the data obtained after gamma radiation.

For the purposes of the performed experiment, NOR memories from different manufacturers were subjected to radiation. Conclusions made from the analysis of experimental results are also valid for NAND flash memories. Therefore, the results are related to the effects of radiation which appear at the level of FG cells, regardless of the specific sequence architecture.

The research was conducted on four types of flash memory, with a capacity of $512 \mathrm{Kbit}$, including Numonyx M25P05-A (marked as Type 1), Atmel AT25F512B (marked as Type 2), SST SST25VF512 (marked as Type 3), and Macronix MX25L5211E (marked as Type 4). Type 1 memory has TSS0P8 packaging, while the other three have SOIC package.

All the tested memory chips are programmed by the scheme called "chessboard," with alternately switching on/off 


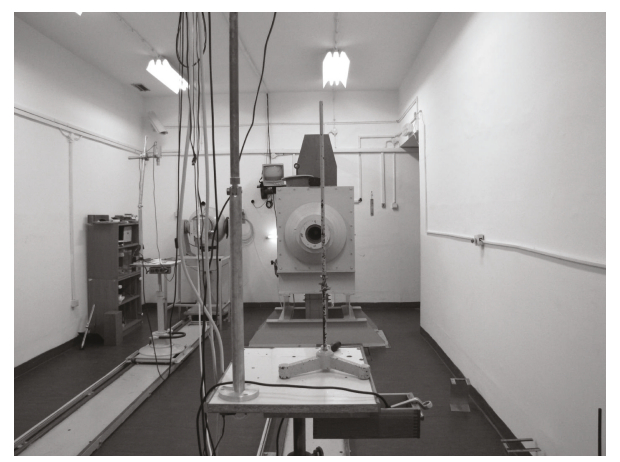

FIgURE 2: Standard Co-60 radiation field used in the Laboratory for Radiation Protection at the Institute of Nuclear Sciences "Vinča."

the cells (alternately writing 1 and 0 ) before radiation, while chip connections remain nonpolarized during the radiation. In this way, the influence of gamma radiation on the memory cells and the ability to keep the stored data is examined.

Radiation was carried out on Co-60 in the Laboratory for Radiation Protection of the Institute of Nuclear Sciences "Vinča" in Belgrade, Serbia. The unit of radiation field is determined by the value of absorbed dose in air at different distances from the source of radiation. The absorbed dose of memory samples is determined by changing the position of the sample in the field during radiation. The absorbed dose in silicon is calculated from the absorbed dose in the air by setting the energy absorption coefficient for the average energy of Co-60, which is exposed to the effects of gamma rays, to the value of $1.25 \mathrm{MeV}$. All measurements were performed at room temperature. Figure 2 shows the photography of the standard radiation field used in the Laboratory for Radiation Protection at the Institute of Nuclear Sciences "Vinča."

Five samples of four different types of memory were subjected to gamma rays. The absorbed dose ranged from $1 \mathrm{kGy}$ to $12 \mathrm{kGy}$, with a rise of $1 \mathrm{kGy}$. The presented results were obtained by averaging all the experimental results for all the five samples. The measurement uncertainty of obtained results was about $10 \%$ for all the samples.

\section{Results and Discussion}

To perform the experiments, NOR memories from different manufacturers were selected. Conclusions are also applicable to the NAND memories because they are related to the effects of radiation on the cell level with a floating gate, regardless of the architecture of the memory matrix.

4.1. TID Effects (Exposure to Gamma Rays). Four types of flash memory programmed in "chessboard" scheme, in which adjacent cells are alternately in "on" and "off" states before radiation, have been analyzed. During radiation, the pins of chips were free. In this way, the influence of gamma rays on the ability to hold the stored data (retention) of tested memory, that is the ability of flash memory to preserve the written content in the absence of the power supply, is examined. The radiation field at the cobalt unit is characterized by values of

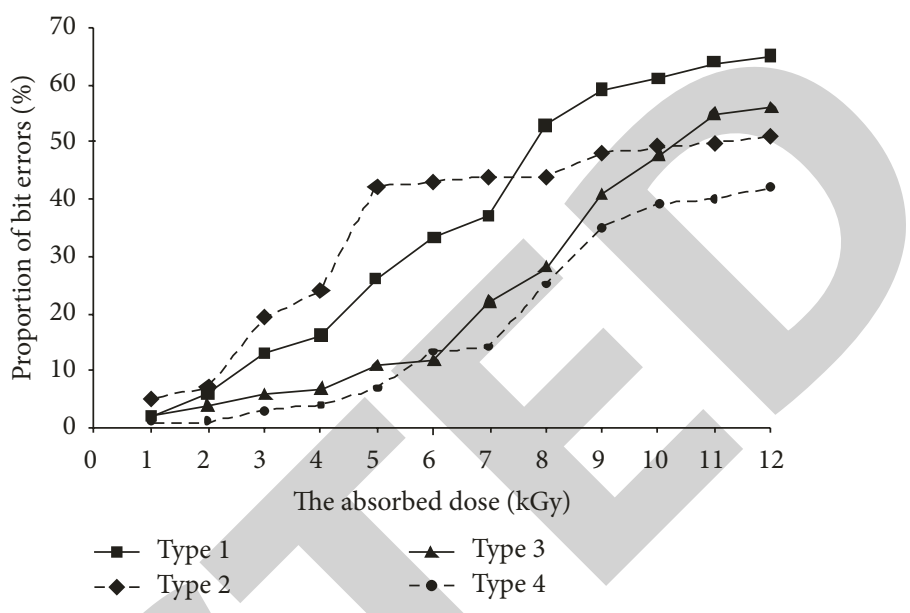

FIGURE 3: Registered percentage of bit errors depending on the absorbed dose for the four analyzed types of flash memory.

the intensity of the absorbed dose in air at different distances from the Co-60 source.

The results presented in Figure 3 were obtained as average values for groups of 5 chips with the measurement uncertainty of results about $10 \%$ for all the groups of chips.

The graph in Figure 3 shows the percentage change in bit errors (i.e., the ratio of the bits number and the disturbance compared to the original written state and the total number of bits, which is 512000) with an increasing absorbed dose, up to a dose of about $9 \mathrm{kGy}$, after which the saturation of the errors percentage occurs. In the memory of Type 2 , a slightly steeper increase in the number of bit errors occurred in a lower dose range than in the other three memories. Specifically, the saturation of Type 2 has occurred at a dose of about $5 \mathrm{kGy}$. The memory of Type 1 showed the highest percentage of bit errors (65\%) at the maximum dose (12 kGy), while the memory of Type 4 had the lowest number of errors (42\%) at the maximum dose. Saturation is the least pronounced in the memory of Type 3 , which means that with this type of memory errors could be increased at doses greater than $12 \mathrm{kGy}$.

The graph in Figure 4 shows the results of recovery for the examined four types of flash memories at room temperature after exposure to a maximum dose of $12 \mathrm{kGy}$. The percentage of bit errors was measured once a week for nine weeks after radiation.

In all the four types of memory, the temperate recovery of the initially written content is observed. By the end of 9 weeks, Type 4 memory had the lowest percentage of the remaining errors (17\%), while the maximum bit errors remained in the memory of Type 2 (32\%).

4.2. Damages Caused by Displacement (Simulation of the Ions Passage). For the numerical simulation of the radiation effects on the films of superinsulators, the Monte-Carlo method is used, based on the stochastic nature of the interactions of radiation with the structural units of the material environment through which the radiation passes. In real 


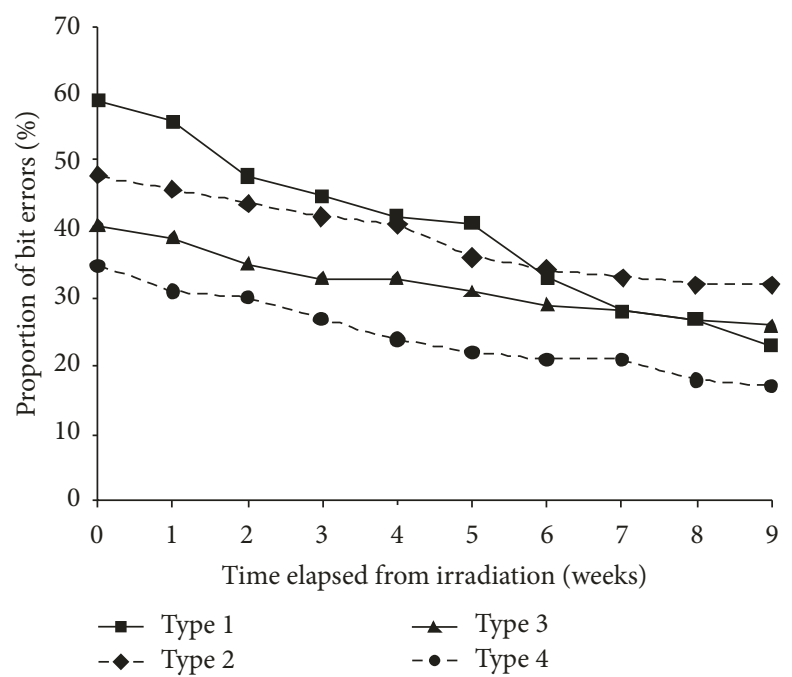

Figure 4: Recovery of the four tested types of flash memory at room temperature for nine weeks after exposure to a maximum dose of $12 \mathrm{kGy}$.

physical experiments, the measurements uncertainty is evaluated and calculated when the experiment ends. Numerical simulations of the same physical process with Monte-Carlo methods can be performed with predetermined uncertainty, following an adequate number of primary particles of radiation, as well as secondary particles produced in the material.

Monte-Carlo simulations of ion beams passing through the material in the superinsulator state are implemented in the TRIM module of the software package SRIM [11]. This program allows the calculation of the energy loss of incident radiation through ionization, photon excitation of grid, and displacement of atom material [12].

An ion beam in the simulations was chosen to match the radiation field in which the electronic components often are ions of hydrogen, helium, lead, then phosphorus, boron, and arsenic. SRIM limits calculations on the monoenergetic beams, but for repeated simulations, energy of each ion species is varied due to the range of values represented in practice.

A simulation of the ions passage is designed to compare the radiation damage by displacing atoms in the dielectric $\mathrm{ONO}$ and tunnel oxide. The dimensions of the layers used in the simulations are given in Figure 5 and taken from [13].

ONO dielectric is presented in the form of a three-layered structure with upper and lower oxide $\left(\mathrm{SiO}_{2}\right)$ and $\mathrm{Si}_{3} \mathrm{~N}_{4}$ layer between them. Simulations were performed with the ion beam, which normally falls on the upper surface of the cells (control gate).

The simulation results are presented in Figures 6, 7, and 8; the unit is on the $x$-axis, which corresponds to a depth within the flash cell. The incident beam in all cases is simulated with $10^{4}$ ions.

Comparing the results in Figures 6 and 7(a), it can be concluded that due to nanometre dimensions, flash memory cells are much less sensitive to the proton effects with $\mathrm{MeV}$

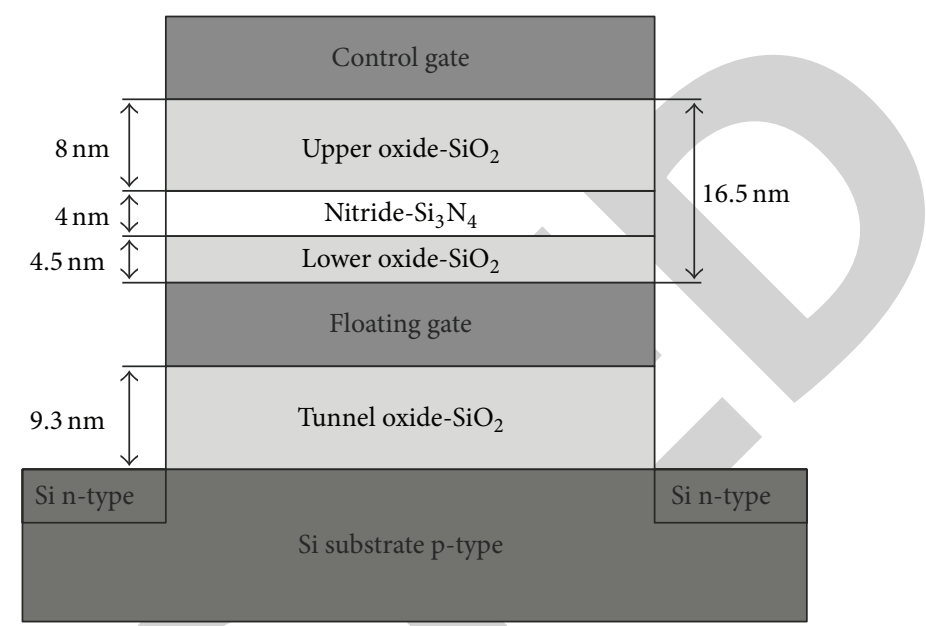

FIGURE 5: Model of the flash memory cell used in the numerical simulation of the ions passage.

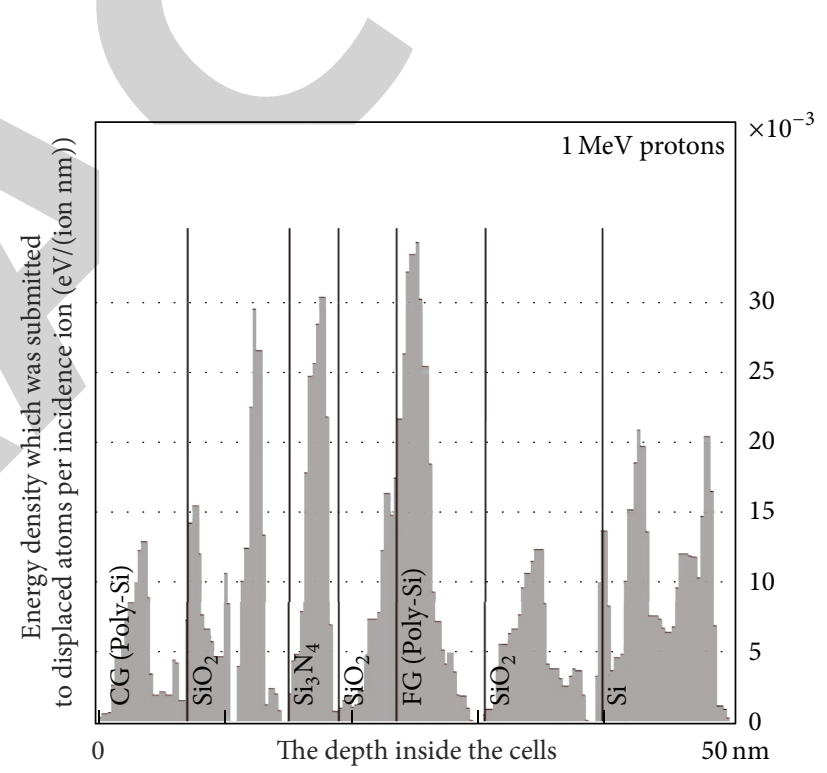

Figure 6: Energy density of incident protons per unit of depth which is submitted to atoms displaced in the layers of the flash cell (expressed per incident proton) for $1 \mathrm{MeV}$ protons.

energies than the protons with energies in the keV field. The energy density of incident protons per unit of depth, which is delivered to atoms displaced in the layers of the flash cell, is much lower for $1 \mathrm{MeV}$ than for $10 \mathrm{keV}$ protons.

The high degree of the flash cell immunity to the effects of particle radiation in $\mathrm{MeV}$ energy field is expressed by the results for $2 \mathrm{MeV}$ alpha particles given in Figure 8 .

The divergence of an alpha particle beam is negligible throughout the entire structure of the cell. Displaced atoms are spatially concentrated, with an almost straight-line path of alpha particles, and the number of lateral branches, in which the secondary displacement occurs, is small (Figure 8(a)). Except on the separated surface of the nitride layer and lower oxide in ONO dielectric, the energy loss of alpha particles due to the displacement of atoms is almost negligible. The results 


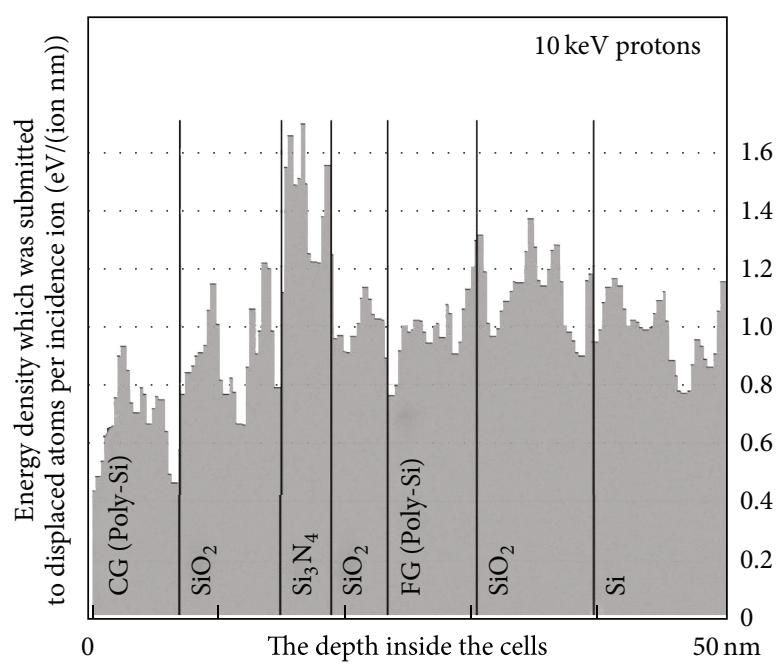

(a)

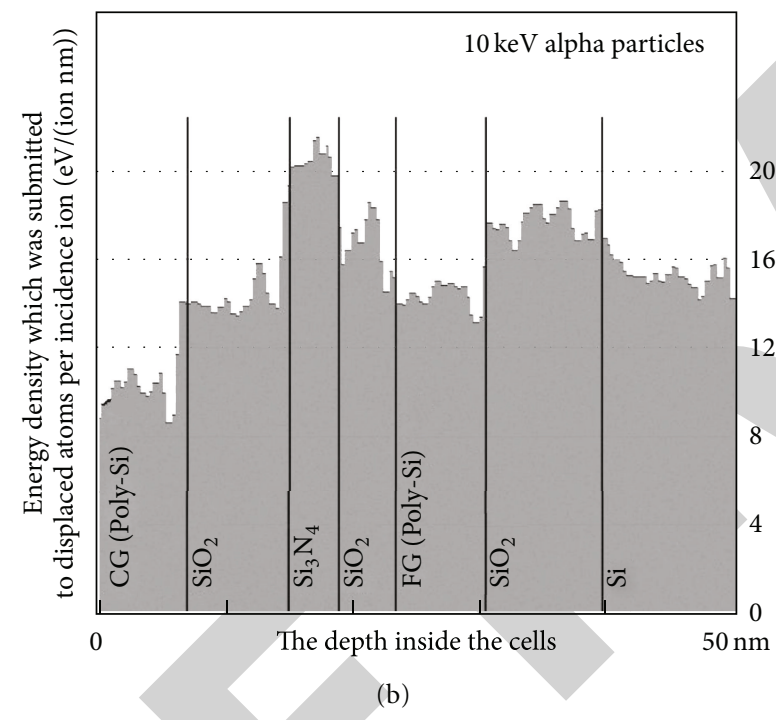

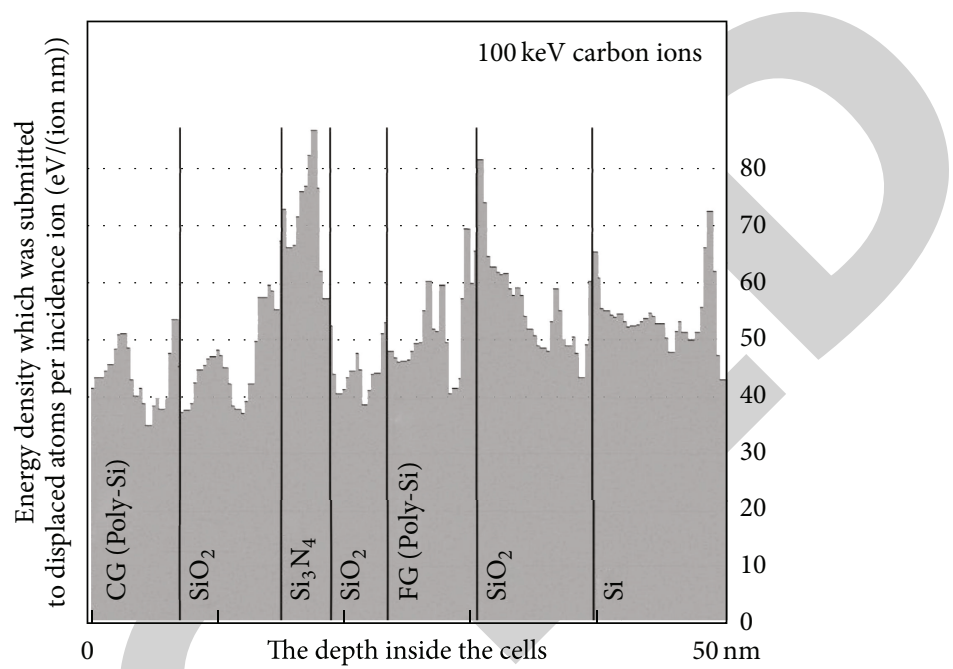

(c)

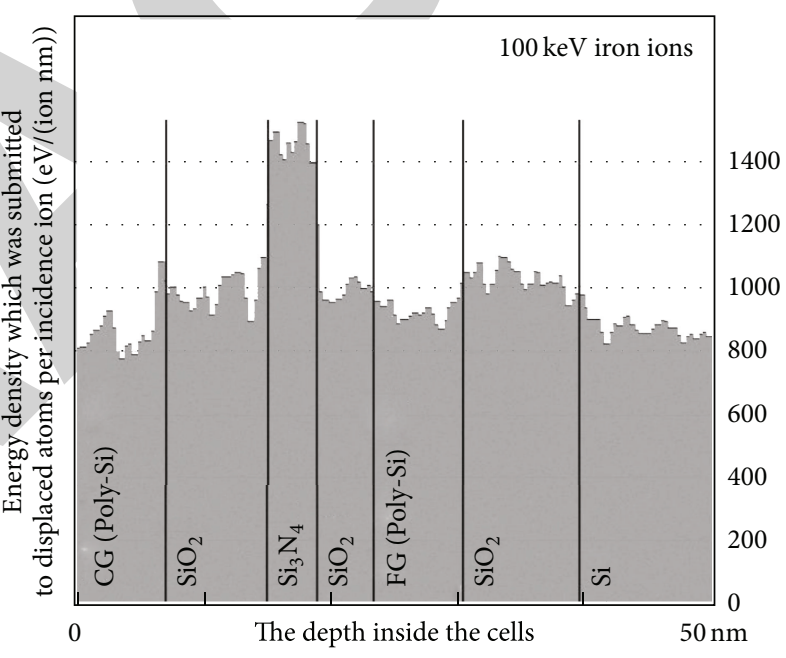

(d)

Figure 7: Density of ion energy (per unit of depth), which was submitted to atoms displaced in the layers of the flash cell (expressed per incident ion) for (a) $10 \mathrm{keV}$ protons, (b) $10 \mathrm{keV} \alpha$ particles, (c) $100 \mathrm{keV}$ ions of C, and (d) $100 \mathrm{keV}$ ions of Fe.

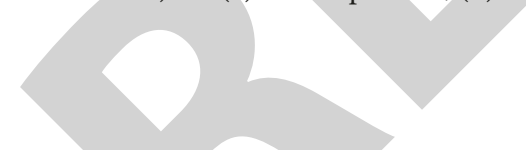

in Figures $8(\mathrm{~b})$ and $8(\mathrm{c})$ show that the density of vacancies produced by the incident beam are much lower for $2 \mathrm{MeV}$ than for $10 \mathrm{keV}$ particles.

\section{Conclusion}

In the analysis of TID effects, the ability of keeping written data of the four models of flash memory after exposure to gamma rays is examined. The increase in the number of bit error rate with increasing the absorbed dose can be attributed to the effects of tunnel oxide radiation and oxide which separates the floating gate in the cell of flash memory. The theoretical analysis of these effects shows that under the influence of radiation, the value of the threshold voltage is reduced for programmed cells, while in the cells that have been erased, it is increased. This means that both states are subject to disturbance which is, while reading the memory, registered as bit errors. The percentage of bit errors did not differ much in the four tested types of memory, which indicates that the sensitivity to gamma rays depends primarily on the thickness of the dielectric layers of cells, which is about the same for all tested memories, since they belong to the same generation of technology.

Partial recovery of errors caused by radiation, in a few weeks at room temperature, was observed in all the four types of memory. This explains recovery with the gradual compensation of charge trapped in the tunnel oxide, with electrons tunneling from the substrate.

An analysis of the effects due to the displacement of atoms was performed using a numerical simulation of the 


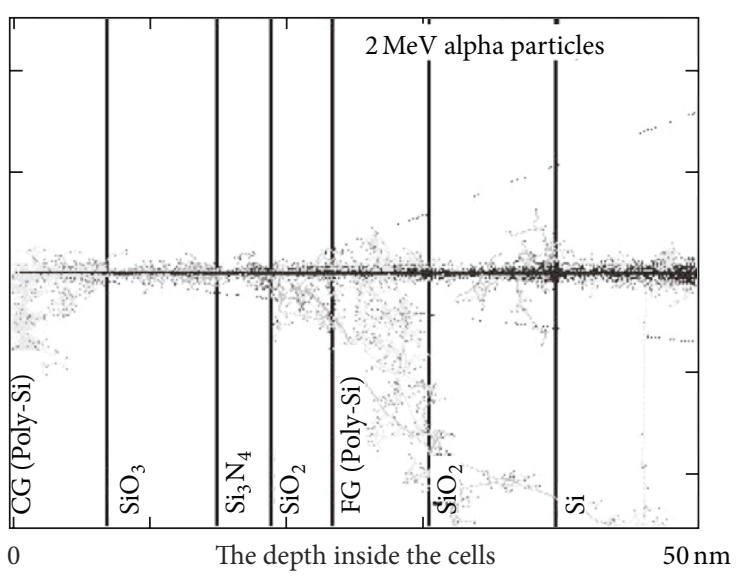

(a)
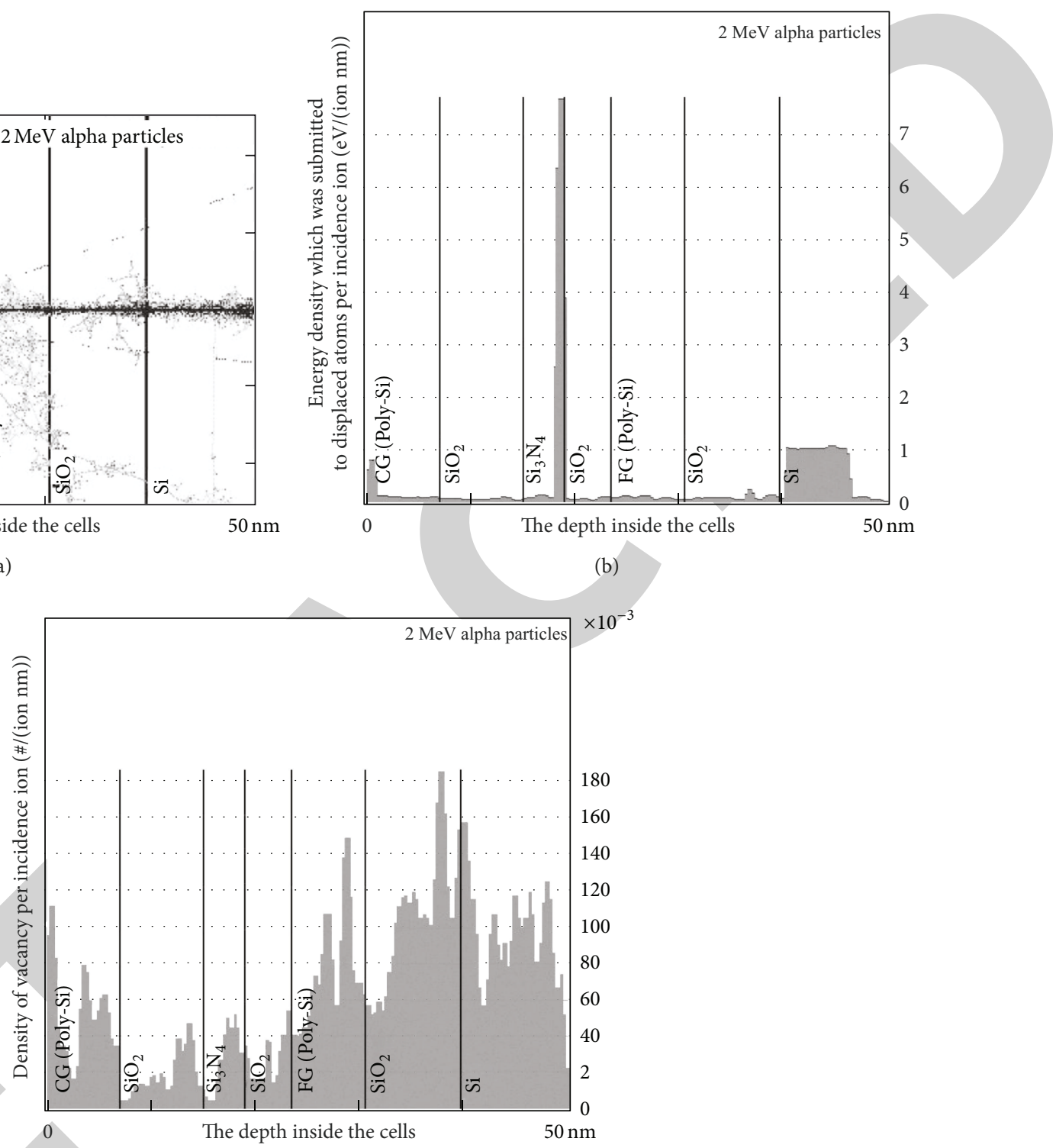

(c)

FIGURE 8: Results for the incident beam of $2 \mathrm{MeV}$ : (a) traces of particles (dark traces correspond to alpha particles, while the lighter traces are the path of the displaced ions $\mathrm{Si}, \mathrm{O}$, and $\mathrm{N}$ ), (b) energy density of alpha particles submitted to displaced atoms, and (c) density of vacancy caused by displacement of atoms.

ions passage through the cell of flash memory. Due to the low thickness, the layers of the flash memory cells are insensitive to ion energies greater than approximately $1 \mathrm{MeV}$. The simulation of radiation transport, however, shows that for a given type and energy of ions in some layers of the cells a considerable number of displaced atoms and vacancies occur. Damages caused by such displacement, which occur in the structure of flash cells, can affect the whole range of its parameters (threshold voltage, leakage current, access time, and time of writing data), as well as preventing the reprogramming of the cell. Some types of errors may occur even for devices that are not biased during the time that heavy ions strike occurs. Error detection and correction may be a viable way to recognize this type of failure mechanism, but it is also necessary to understand how and why the errors are generated within the device, as well as whether internal errors in the memory controller will affect their operation in the space.

\section{Acknowledgment}

The Ministry of Education, Science and Technological Development of the Republic of Serbia supported this work under Contract 171007. 


\section{References}

[1] M. Vujisić, K. Stanković, E. Dolićanin, and P. Osmokrović, "Radiation hardness of COTS EPROMs and EEPROMs," Radiation Effects and Defects in Solids: Incorporating Plasma Science and Plasma Technology, vol. 165, no. 5, pp. 362-369, 2010.

[2] J. E. Velazquez-Perez and Y. G. Gurevich, "Charge-carrier transport in thin film solar cells: new formulation," International Journal of Photoenergy, vol. 2011, Article ID 976063, 2011.

[3] S. Sladić, S. Skok, and D. Nedeljković, "Efficiency considerations and application limits of single-phase active power filter with converters for photoenergy applications," International Journal of Photoenergy, vol. 2011, Article ID 643912, 2011.

[4] M. Vujisić, K. Stanković, and P. Osmokrović, "A statistical analysis of measurement results obtained from nonlinear physical laws," Applied Mathematical Modelling, vol. 35, no. 7, pp. 31283135, 2011.

[5] Holmes-Siedle and L. Adams, Handbook of Radiation Effects, Oxford Science Publication, Oxford, UK, 1993.

[6] G. C. Messenger and M. S. Ash, The Effects of Radiation on Electronic Systems, Van Nostrand Reinhold, New York, NY, USA, 2nd edition, 1991.

[7] K. D. Stanković, "Influence of the plain-parallel electrode surface dimensions on the type a measurement uncertainty of gm counter," Nuclear Technology and Radiation Protection, vol. 26, no. 1, pp. 39-44, 2011.

[8] M. Zdravković, A. Vasić, R. Radosavljević, M. Vujisić, and P. Osmokrović, "Influence of radiation on the properties of solar cells," Nuclear Technology \& Radiation Protection, vol. 26, no. 2, pp. 158-163, 2011.

[9] R. Radosavljević and A. Vasić, "Effects of radiation on solar cells as photovoltaic generators," Nuclear Technology \& Radiation Protection, vol. 27, no. 1, pp. 28-32, 2012.

[10] M. Vujisić, N. Marjanović, I. Fetahović, K. Stanković, and P. Osmokrović, "Influence of radiation on titanium dioxide mermistors," Scientific Publications of the State University of Novi Pazar Series A: Applied Mathematics, Informatics \& Mechanics, vol. 4, no. 1, pp. 75-82, 2012.

[11] J. F. Ziegler, J. P. Biersack, and M. D. Ziegler, "SRIM (The Stopping and Range of Ions in Matter)," http://www.srim.org/.

[12] J. R. Srour, C. J. Marshall, and P. W. Marshall, "Review of displacement damage effects in silicon devices," IEEE Transactions on Nuclear Science, vol. 50, no. 3, pp. 653-670, 2003.

[13] A. Gasperin, A. Paccagnella, G. Ghidini, and A. Sebastiani, "Heavy ion irradiation effects on capacitors with $\mathrm{SiO}_{2}$ and ONO as Dielectrics," IEEE Transactions on Nuclear Science, vol. 56, no. 4, pp. 2218-2224, 2009. 